\title{
ARTICLE
}

Acute myeloid leukemia

\section{A transcriptomic continuum of differentiation arrest identifies myeloid interface acute leukemias with poor prognosis}

\author{
Jonathan Bond ${ }^{1,2,3,4} \cdot$ Aleksandra Krzywon ${ }^{5,6,7,8} \cdot{\text { Ludovic Lhermitte } \mathbb{1}^{1,2} \cdot \text { Christophe Roumier }^{9,10} \cdot \text { Anne Roggy }}^{1,2,11}$. \\ Mohamed Belhocine ${ }^{1,2}$. Alexander Abdulkader Kheirallah ${ }^{5,6} \cdot$ Patrick Villarese $^{1,2} \cdot$ Guillaume Hypolite $^{1,2}$. \\ Francine Garnache-Ottou $^{11} \cdot$ Sylvie Castaigne ${ }^{12} \cdot$ Nicolas Boissel $^{13} \cdot$ Vahid Asnafi $^{1,2} \cdot$ Claude Preudhomme $^{9,10}$. \\ Hervé Dombret $^{13}$ • Elisa Laurenti (iD ${ }^{5,6}$. Elizabeth Macintyre (D) $^{1,2}$
}

Received: 18 November 2019 / Revised: 11 June 2020 / Accepted: 1 July 2020 / Published online: 13 July 2020

(c) The Author(s) 2020. This article is published with open access

\begin{abstract}
Classification of acute lymphoblastic and myeloid leukemias (ALL and AML) remains heavily based on phenotypic resemblance to normal hematopoietic precursors. This framework can provide diagnostic challenges for immunophenotypically heterogeneous immature leukemias, and ignores recent advances in understanding of developmental multipotency of diverse normal hematopoietic progenitor populations that are identified by transcriptional signatures. We performed transcriptional analyses of a large series of acute myeloid and lymphoid leukemias and detected significant overlap in gene expression between cases in different diagnostic categories. Bioinformatic classification of leukemias along a continuum of hematopoietic differentiation identified leukemias at the myeloid/T-lymphoid interface, which shared gene expression programs with a series of multi or oligopotent hematopoietic progenitor populations, including the most immature CD34+CD1a-CD7 - subset of early thymic precursors. Within these interface acute leukemias (IALs), transcriptional resemblance to early lymphoid progenitor populations and biphenotypic leukemias was more evident in cases originally diagnosed as AML, rather than T-ALL. Further prognostic analyses revealed that expression of IAL transcriptional programs significantly correlated with poor outcome in independent AML patient cohorts. Our results suggest that traditional binary approaches to acute leukemia categorization are reductive, and that identification of IALs could allow better treatment allocation and evaluation of therapeutic options.
\end{abstract}

Supplementary information The online version of this article (https:// doi.org/10.1038/s41375-020-0965-z) contains supplementary material, which is available to authorized users.

Elisa Laurenti

el422@cam.ac.uk

$\triangle$ Elizabeth Macintyre

elizabeth.macintyre@aphp.fr

1 Université de Paris, Institut Necker Enfants Malades (INEM), Institut national de la santé et de la recherche médicale (INSERM UMR1151), 75743 Paris, France

2 Laboratory of Onco-Hematology, Assistance Publique-Hôpitaux de Paris (AP-HP), Hôpital Necker-Enfants Malades, Paris, France

3 Systems Biology Ireland, School of Medicine, University College Dublin, Dublin, Ireland

4 National Children's Research Centre, Children's Health Ireland at Crumlin, Dublin, Ireland

5 Department of Haematology, University of Cambridge, Cambridge, United Kingdom
6 Wellcome and MRC Cambridge Stem Cell Institute, University of Cambridge, Cambridge, United Kingdom

7 Silesian University of Technology, Department of Systems Biology and Engineering, Gliwice, Poland

8 Maria Sklodowska-Curie National Research Institute of Oncology, Gliwice Branch, Gliwice, Poland

9 Cancer Research Institute, INSERM Unité Mixte de Recherche (UMR)-S 1172, F-59000 Lille, France

10 CHU Lille, Laboratory of Hematology, F-59000 Lille, France

11 Univ. Bourgogne Franche-Comté, INSERM, EFS BFC, UMR1098, Interactions Hôte-Greffon-Tumeur/Ingénierie Cellulaire et Génique, F-25000 Besançon, France

12 Hematology/Oncology, Versailles Hospital and Versailles Saint Quentin University, Le Chesnay, France

13 Université Paris Diderot, Institut Universitaire d'Hématologie, EA-3518, AP-HP, University Hospital Saint-Louis, Paris, France 


\section{Introduction}

Successful management of acute leukemia is underpinned by accurate diagnostic classification, which provides a basis for treatment allocation, risk stratification and implementation of targeted therapies [1]. Although knowledge of the molecular landscape of leukemia has increased enormously over the past decades, contemporary classification remains heavily predicated on simple immunophenotypic resemblance to either myeloid or lymphoid normal hematopoietic precursors [2]. While this system has historically been successful, some leukemia categories provide specific diagnostic and therapeutic challenges. The current World Health Organization (WHO) classification [2] recognizes acute leukemias of ambiguous lineage that either lack lineage-specific markers (acute undifferentiated leukemias, AUL) or express a mixture of myeloid and lymphoid antigens (mixed phenotype acute leukemias, MPAL). There is little consensus on the best treatment approaches for these patients, and prognosis is usually poor [3-5].

This framework also poses difficulties for some cases of T-acute lymphoblastic leukemia (T-ALL) and acute myeloid leukemia (AML). T-ALL can be subclassified by immunogenotypic and phenotypic resemblance to either immature/ early thymic precursor (ETP), early cortical or late cortical normal T-progenitor equivalents [6, 7]. However, the genotypic and phenotypic heterogeneity of immature T-ALLs mean that robust biological classification of this group is not straightforward [8]. A subset of these cases harbor mutations that are also commonly seen in AML, suggesting that at least some immature T-ALLs may arise from transformation of a bipotent lympho-myeloid progenitor [9-13]. In addition, diagnostic distinction from AML by immunophenotype is often not clear-cut, as immature T-ALLs commonly express myeloid lineageassociated markers [14]. Conversely, the most phenotypically immature AML subgroup, M0-AML, is also biologically heterogeneous and expresses lymphoid-associated antigens such as CD7 or TdT in about 50\% of cases [15]. Immature T-ALLs are frequently chemoresistant and require intensive treatment $[10,14,16]$, while M0-AML cases have poor outcomes compared to other AML subgroups [17, 18], so it is clinically important to consider whether improved classification of these cases might allow better therapeutic choices.

Current leukemia classification also takes little account of modern advances in understanding of human hematopoiesis, and the recognition of a diverse range of pluri- and multipotent progenitors, as identified by transcriptional signatures and functional assays [19]. In particular, traditional notions of an early lymphoid/myeloid dichotomy have been undermined by the discovery of a multitude of lymphoid committed cell types which retain myeloid potential at different stages of differentiation: within the phenotypic stem cell [20] or progenitor compartment [21-25] and in the thymus [26, 27]. The relevance of these cell types in the context of leukemia is only beginning to be explored [22, 28].

Leukemic transcriptome profiling should help to improve categorization, but traditional analytical approaches have their shortcomings. T-ALL can be reproducibly categorized according to a limited number of expression signatures that correlate with the phenotype of differentiation arrest $[6,29,30]$. Data may also be interrogated by gene set enrichment analysis (GSEA), which has revealed that immature/ETP-ALLs transcriptionally resemble both normal hematopoietic stem cell (HSC) and immature myeloid precursors [9]. However, these approaches rely on comparisons of predefined sample groups, neglect transcriptional heterogeneity of individual leukemias in each group and cannot resolve relationships between groups. These analyses therefore provide limited information about the spectrum of differentiation arrest in acute leukemia.

Evolutions in genomic analytical methods provide an opportunity to refine leukemia classification. We have analyzed a series of acute leukemias that comprised a high proportion of immature T-ALLs and AMLs using several complementary methods. Firstly, we show that hierarchical clustering and dimensionality reduction approaches consistently identify an AML-like subset of T-ALL. To gain deeper insight into the spectrum of differentiation arrest in these leukemias, we then used the novel Iterative Clustering and Guide Gene Selection method (ICGS). This technique, when applied to single-cell RNAsequencing data, has been shown to infer cellular states from transcriptional data, identify modules of guide genes that are specific to these cellular developmental states in an unbiased, agnostic manner, and infer developmental relationships between these states [31]. We show that application of ICGS to global expression data identifies a continuum of differentiation arrest, which includes a group of myeloid/ T-lymphoid interface leukemias that lack clear lineage identity, and which respond poorly to AML treatment regimens.

\section{Methods}

\section{Microarray data analysis}

All computational analysis was performed in R (v.3.3.2 or above) unless otherwise specified. Data were normalized with normalize.quantiles function from the preprocessCore v1.34.0 package and batch effects between 2 independent arrays were corrected using the ComBat function (sva 
a

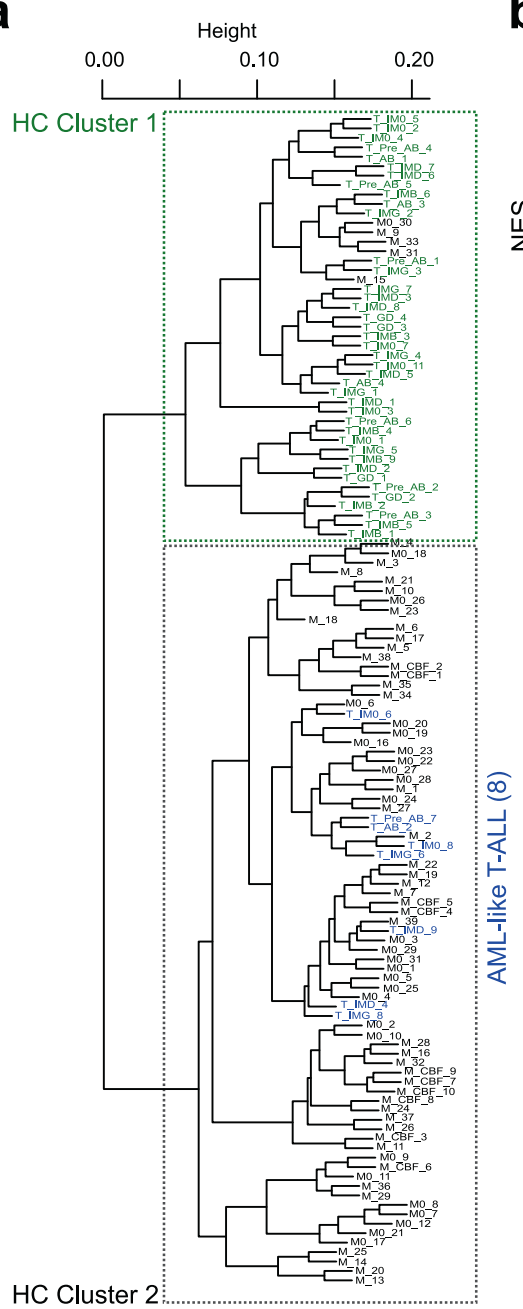

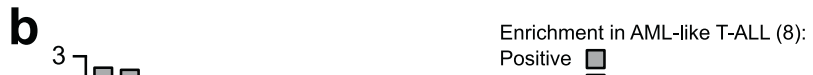

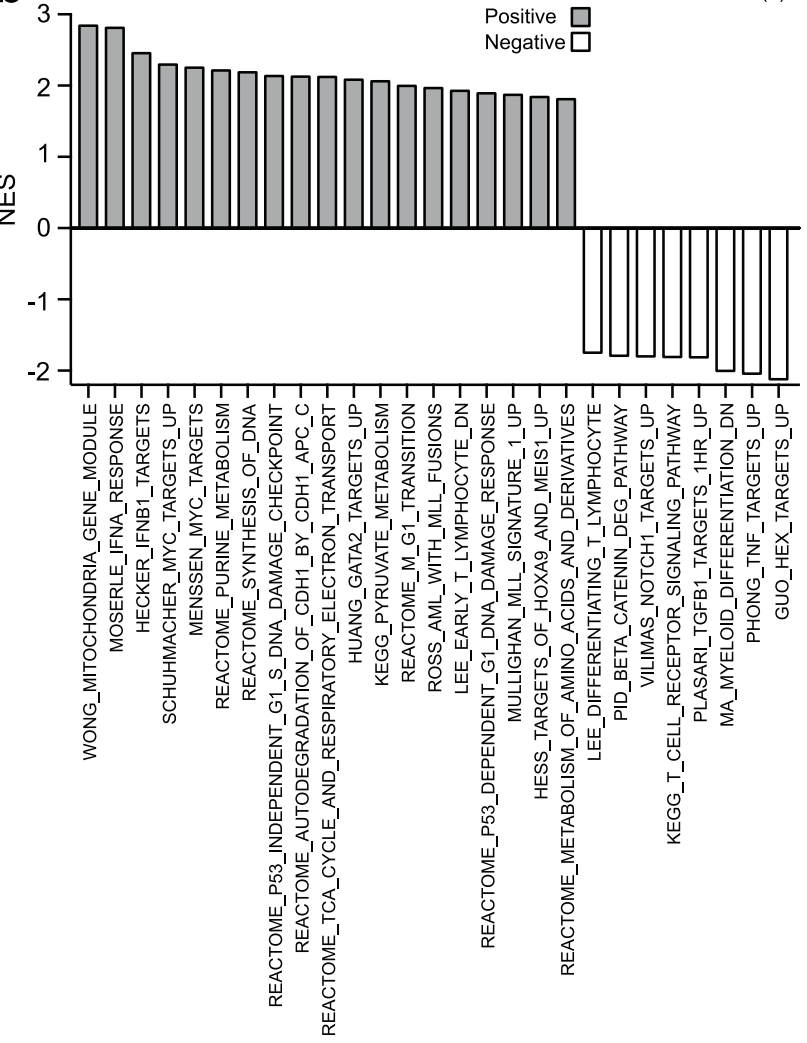

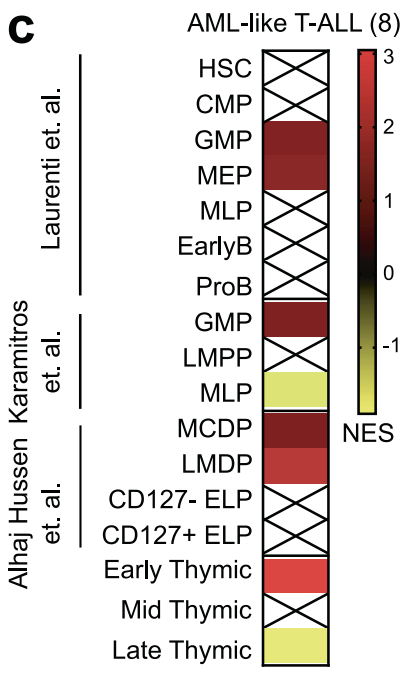

d $\square T-A L L$

- AML-like T-ALL (8)
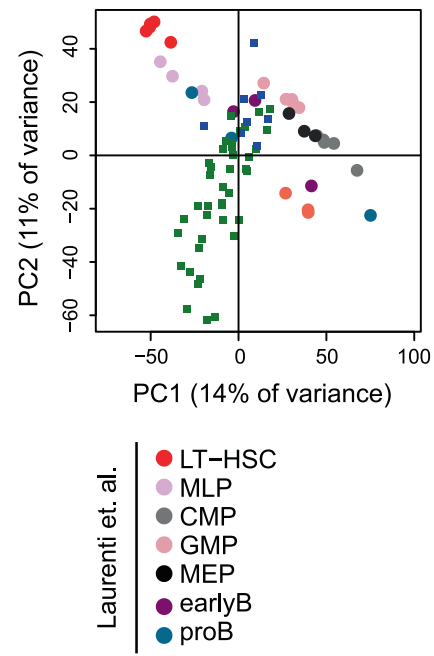

package). Hierarchical clustering was performed with the hclust function with distance (1-Pearson correlation) and complete clustering method. Principal Component
Analysis (PCA) was performed with prcomp function. Both hierarchical clustering and PCA were performed on all probes. 
Fig. 1 Transcriptional profiling identifies AML-like T-ALLs that are enriched for immature myeloid and thymic progenitor transcriptional signatures. a Unsupervised hierarchical clustering (HC) of the transcriptional profiles of 124 acute leukemias, comprising $48 \mathrm{~T}$ ALLs and 76 AMLs. A subset of T-ALL cases segregates with the AML cluster. b GSEA analysis of pathways significantly enriched in AML-like T-ALLs vs the rest of the T-ALL cohort. The MSigDB C2 collection of genesets was used and only selected genesets with FDR $<0.05$ are shown. NES normalized enrichment score. $\mathbf{c}$ Enrichment of selected normal hematopoietic progenitor transcriptional signatures derived from the indicated published datasets or our own analysis of thymic subpopulations (gene-sets provided in Supplementary Table S1) in AML-like T-ALLs by GSEA. NES normalized enrichment score, crossed out boxes indicate gene-sets that are not significantly enriched (FDR > 0.05). HSC hematopoietic stem cell, CMP common myeloid progenitor, GMP granulocyte-monocyte progenitor, MEP megakaryocytic-erythroid progenitor, MLP multi-lymphoid progenitor, LMPP lymphoid-primed multipotent progenitor, MDCP monocyte-dendritic cell progenitor, LMDP lymphoid-mono-dendritic progenitor, ELP early lymphoid precursor. d 2D PCA map of umbilical cord blood stem and progenitor populations and T-ALL gene expression patterns [38]; distribution of AML-like T-ALLs (blue squares) is significantly different to that of other T-ALLs (PC1: $p=$ 0.003; PC2: $p=4.1 \times 10^{-5}$ by two-sided t-test).

\section{ICGS}

ICGS was performed with AltAnalyze software v.2.1.0 (http://www.altanalyze.org/) using HOPACH clustering, with default settings for gene expression analysis options (moderated t-test for group comparison and Benjamini - Hochberg false discovery rate $<0.05$ ). The gene expression filtering option was set to 2 . Cell cycle genes were excluded using the most stringent parameter. From the Liu et al. pediatric cohort [32], all samples were used, whereas from the Chen et al. cohort [33] only adult samples ( $>18$ years) were selected. Heatmap visualization of ICGS data was performed in AltAnalyze.

\section{Differential expression analysis}

Differentially expressed genes were derived using the limma package (lmFit function) for microarray and DESeq2 for RNA-Seq. Contrast matrices between selected groups are listed in Supplementary Table S1. Genes were considered differentially expressed if Benjamini-Hochberg false discovery rate $(\mathrm{FDR})<0.05$. Gene ranking for Gene Set Enrichment Analyis (GSEA) was performed according to $t$-statistic for microarray data or Wald statistic for RNAseq data. For the thymic subpopulation dataset, most variable genes across all populations were selected as the union of all the probes differentially expressed between any two populations (thymic HVGs, 8751 probes).

\section{Pathway and gene set enrichment analysis}

GSEA was performed with GSEA software (http://software. broadinstitute.org/gsea/index.jsp) using the C2.all.v6.1 collection of genesets from MSigDB (http://software.broa dinstitute.org/gsea/msigdb/index.jsp) or a collection of custom genesets (Supplementary Table S1) derived from datasets generated here or publicly available [19, 23, 24, 34-36]. When specific genesets were derived from published data, differential expression analysis was performed as indicated above using the contrasts indicated in Supplementary Table S1. Differentially expressed genes were then ranked by $t$-statistic for microarray data or by Wald statistic for RNA-seq data and the top 500 genes (or all genes with FDR $<0.05$ if $<500$ genes had FDR $<0.05$ ) were selected as genesets to be tested by GSEA. GSEA outputs were either visualized with the EnrichmentMap plugin (FDR Q-value cutoff 0.05) of Cytoscape (v.3.2.0), or with heatmaps generated with Prism software (v.7). ClueGO analysis was performed with the ClueGO plugin (v.2.1.6) of Cytoscape (v.3.2.0), using the GO Term Fusion option and otherwise default parameters.

Code pertaining to each analysis is available upon request.

\section{Results}

\section{Transcriptional profiling identifies an AML-like subset of T-ALL}

We performed transcriptional profiling of a series of 124 acute T-lymphoid and myeloid leukemias (See Supplementary Methods). In order to maximize our ability to detect shared patterns of gene expression in immature leukemias, the study cohort was intentionally skewed to include a higher proportion of these cases than would be observed by chance. 26/48 TALLs (54.2\%) had an immature T-receptor immunogenotype [37], comprising 9 IM0 (germline TR), 9 IMD (TRD rearrangement only) and 8 IMG (TRG and TRD rearranged but absent or incomplete TRB rearrangement) leukemias. Similarly, 28/76 AML samples (40.8\%) were categorized as M0AML. Patient details are shown in Supplementary Table S2.

Unsupervised hierarchical clustering (HC) analysis of the expression data revealed that T-ALL and AML samples largely formed two distinct groups (HC cluster 1 and $\mathrm{HC}$ cluster 2, Fig. 1a). Strikingly, 8/48 T-ALLs (16.7\%, henceforth 'AML-like T-ALL') segregated in the AML cluster in this unsupervised analysis, and clustered together when HC was restricted to T-ALLs (Supplementary Fig. S1A). When visualized by Principal Component Analysis (PCA), T-ALL and AML samples were distributed differently along the first principal component. Notably, T-ALL samples clustering with AMLs by HC overlapped with AML samples (Supplementary Fig. 1B).

Not all of these AML-like T-ALLs exhibited immunogenotypic immaturity (6/8) or had an ETP-ALL 
immunophenotype (4/7 fully-phenotyped samples) [14], indicating that AML-like transcription features are not restricted to previously identified categories of less differentiated T-ALLs.

\section{AML-like T-ALLs are enriched for immature myeloid and thymic progenitor transcriptional signatures}

We next examined the transcriptional differences between AML-like cases and the rest of the T-ALL cohort. 2274 genes (Supplementary Table S3) were significantly differentially expressed between the groups (FDR $<0.05)$, with 1213 and 1061 respectively upregulated and downregulated in AML-like T-ALLs. Pathway analysis revealed that AML-like T-ALLs had elevated expression of genes involved in cell cycle and mitochondrial, amino-acid and pyruvate metabolism, and high levels of interferon-related genes, MYC, HOXA, MEIS1 and GATA2 targets (Fig. 1b). Gene-sets that were previously reported to be upregulated in AML in independent datasets were also significantly overrepresented. In contrast, TCR, NOTCH1 and TNF signaling were all downregulated.

We then sought to better characterize AML-like T-ALLs similarity to normal stem and progenitor cells, firstly by performing GSEA using normal umbilical cord blood (UCB) hematopoietic progenitor transcriptional signatures that we previously reported [38]. AML-like T-ALLs were significantly enriched for megakaryocytic-erythroid progenitor (MEP) and granulocyte-monocyte progenitor (GMP), but not hematopoietic stem cells (HSC) signatures. These leukemias were also enriched for a GMP signature from an independent dataset [23], and resembled lymphoidmono-dendritic progenitors (LMDP) from an UCB-derived humanized murine model of early lymphoid development [24] (Fig. 1c). To confirm transcriptional similarity to myeloid progenitors, we combined the gene expression of the T-ALL samples with that of highly purified stem and progenitor populations [38] on a 2D PCA map. Consistent with the GSEA results, AML-like T-ALLs localized in the HSPC differentiation space, near GMPs (Fig. 1d).

We then performed transcriptional profiling of six phenotypically defined $\mathrm{T}$-lymphoid progenitor groups isolated from a series of human thymuses (Supplementary Fig. S2A). The genes most differentially expressed in each subpopulation (Supplementary Fig. S2B and Supplementary Table S4) were consistent with known T-lymphopoietic transcriptional patterns. PCA also reflected this developmental progression (Supplementary Fig. S2C), which was similar to an in-vitro system of human thymocyte differentiation from UCB CD34+ cells [39] (Supplementary Fig. S2D).

PCA identified 3 main clusters: a rare (Supplementary Fig. S2A) 'early' thymic group comprising CD34+ CD1a-CD7- samples, a 'middle' thymic group comprising
$\mathrm{CD} 34+\mathrm{CD} 1 \mathrm{a}-\mathrm{CD} 7+, \quad \mathrm{CD} 34+\mathrm{CD} 1 \mathrm{a}+$ and $\mathrm{CD} 4+\mathrm{ISP}$ samples and a 'late' thymic group encompassing the transcriptionally similar CD4+CD8+DP/TRLow and CD4+CD8+DP/TRHigh samples. We derived specific gene expression signatures for each of these clusters and used these in GSEAs to assess the transcriptional similarity of AML-like T-ALLs to normal thymocyte subsets. Strikingly, AML-like T-ALLs were strongly positively enriched for genes that were specifically expressed by the most immature CD34+CD1a-CD7 - thymic subpopulation (Fig. 1c). Of note, this signature differed from an ETP transcriptional profile that we previously reported, which was derived by comparison to UCB stem and progenitor cells [38] (Supplemental Fig. 2E-G). Conversely, when compared with the rest of the T-ALL cohort, AML-like T-ALL samples were negatively enriched for 'late' thymic discriminating genes (Fig. 1c). Taken together, these results indicate that AMLlike T-ALLs share gene expression programs with both UCB-derived myeloid-competent progenitors and the most immature thymic precursors, which also retain myeloid differentiation potential [27].

\section{Iterative clustering and guide gene selection analysis identifies a continuum of leukemic differentiation arrest}

We next sought to gain a deeper insight into the spectrum of differentiation arrest in these acute leukemias, by using the recently described ICGS method. This analysis employs serial iterative clustering with pattern-specific guide genes to define coherent transcriptional patterns between samples and then groups these samples into cellular states that recapitulate developmental trajectories [31].

To test the feasibility of applying this approach to leukemic datasets, we initially used ICGS to analyze two published series of adult [33] and pediatric [32] T-ALL. For both cohorts, the ICGS algorithm unbiasedly identified guide gene modules enriched for human stem and progenitor cells (HSPCs, CD34+), myeloid cells and thymocytes (Supplementary Fig. S3A and C and Supplementary Table S5), and ordered the T-ALL samples in clusters along a continuum of expression of these genes. Along this spectrum, adult T-ALLs attributed to ICGS clusters with the lowest expression of thymic-associated genes (Groups A and B), but with high expression of HSPC and myeloid genes, were enriched for the ETP-ALL immunophenotype [10, 12-14]. For the pediatric cohort [32], ICGS ordering recapitulated in an unsupervised manner the classification the authors had derived linking mutations to thymic developmental stages (Supplementary Fig. S3C and D). We thus concluded that ICGS allows unbiased classification of leukemic samples according to their stage of differentiation arrest. 



We then used ICGS to analyze our patient cohort. ICGS classified these leukemias into five developmental clusters that were defined by the levels of expression of a limited number of guide genes (Fig. 2a and Supplementary Table S5) that again predominantly comprised transcripts that discriminate hematopoietic cell types. The proportions 
Fig. 2 Iterative Clustering and Guide Gene Selection (ICGS) analysis identifies a continuum of leukemic differentiation arrest. a, b ICGS analysis of adult and pediatric T-ALLs ( $n=48$ samples) and AMLs ( $n=76$ samples) identifies 5 acute leukemia clusters (top). a Heatmap of expression of guide genes selected by ICGS. Columns represent individual samples. Bars on the top identify ICGS clusters. Rows represent genes, and bars on the side represent blocks of correlated genes. Selected enriched gene ontology groups are shown. Full gene lists are provided in Supplementary Table S5. Leukemic phenotypes are indicated in the bars below the heatmap. b Proportions of leukemic phenotypic groups in each ICGS cluster. c Mutations observed in T-ALL $(n=34)$ and AML $(n=45)$ samples ordered according to ICGS analysis in a. Only mutations found in at least two samples are shown.

of different leukemic phenotypes within each cluster are shown in Fig. 2b. Cluster 1 was defined by high expression of thymic- and lymphoid-related genes (e.g., TCF7, LCK, $B C L 11 B$ ), and comprised T-ALL cases exclusively. Conversely, Clusters 4 and 5 were effectively restricted to AML cases, with concentration of Core Binding Factor (CBF)AMLs in cluster 5. These clusters exhibited increased expression of factors that define myeloid transcriptional modules (e.g., $M P O, C E B P E, C S F 3 R$ ). The intermediate Clusters 2 and 3 were characterized by heterogeneous guide gene expression, and included one third of T-ALL cases (16/48, 33.3\%). Notably, the most immature M0 subtype AMLs were predominantly found in these two clusters (24/ $28,85.7 \%)$, as compared with $14 / 48$ (29.2\%, $p<0.001$ by Fisher test) of non-M0-AML. Also, virtually all AML-like T-ALL samples that were defined by $\mathrm{HC}(7 / 8,87.5 \%)$ were found in either Cluster $2(n=4)$ or $3(n=3)$. ICGS therefore provides a means of classifying leukemias along a spectrum of hematopoietic ontogeny, which in our cohort included a significant number of cases at the interface between T-lymphoid and myeloid lineages. Broadly, these 'interface' acute leukemias (IAL) either showed no clear evidence of mature T-lymphoid or mature myeloid identity (Cluster 2), or had a partial HSPC/mature myeloid signature (Cluster 3).

To test whether ICGS clustering patterns correlated with mutational genotype, we performed targeted next generation sequencing (NGS) of the 79/124 cases (34 T-ALLs and 45 AMLs) where diagnostic material was available. NGS panel details are shown in Supplementary Table S6, comprehensive results are in Supplementary Table S7, and all mutations detected in $\geq 2$ patients are shown in Fig. $2 c$. We found that the spectrum of differentiation arrest defined by ICGS correlated only partially with expected somatic mutation patterns. For example, Cluster 1 was enriched both for T-ALL type NOTCH pathway-activating mutations ( $p<$ 0.0001 , all comparisons by Fisher test) and for mutations that are more frequent in immature T-ALLs [9, 10, 40], e.g., SUZ12 $(p=0.004)$, WT1 $(p=0.0044)$ and IL7R/JAK/ STAT pathway members $(p=0.0364)$.
Similarly, interface clusters were enriched for alterations in DNA methylating factors that are typical of less differentiated leukemias [13, 41, 42], with DNMT3A, IDH1 and IDH2 mutated T-ALLs being confined to cluster 2 ( $p=$ 0.0267 ), but PTEN mutations that normally segregate with phenotypically mature leukemias [43] were significantly more common in AML-like T-ALLs than in other T-ALL cases $(50 \%$ v $7.1 \%, p=0.0287)$. Of note, $R U N X 1$-mutated AMLs were exclusive to interface clusters 2 and 3 ( $p=$ $0.0015)$.

\section{ICGS identifies myeloid leukemias with early lymphoid transcriptional signatures}

Having found that ICGS permits classification of acute leukemias along a spectrum of hematopoietic differentiation, we went on to more precisely characterize the transcriptional identity of individual clusters by GSEA. Analysis of the two independent T-ALL cohorts [32, 33] revealed that the least differentiated clusters were enriched for transcriptional signatures from a series of immature myeloid and lymphoid progenitor populations, in addition to HSCs (Supplementary Fig. S3F).

Within our cohort, Cluster 1 T-ALLs were strongly enriched for mid- and late-thymic expression profiles, and negatively enriched for both early thymic and UCB HSC and myeloid progenitor signatures. AMLs in Clusters 4 and 5 had broadly converse patterns of positive and negative enrichment (Fig. 3a).

Transcriptional differences in IAL Clusters 2 and 3 were less clear-cut. Cluster 2 IALs were enriched for both HSC and a series of lymphoid progenitor signatures, including MLP, LMDP, early B-cell progenitors, T-oriented CD127Early Lymphoid Precursors (ELPs) and CD34+ CD1a-CD7- early thymic cells (Fig. 3a). Cluster 3 IAL cases were more likely to be enriched for myeloid profiles (MEP, GMP and UCB-derived monocyte-dendritic cell progenitors, MDCP), but also showed transcriptional resemblance to several lymphoid subpopulations, including LMDP and both early and mid-thymic signatures (Fig. 3a).

We considered whether this heterogeneity might be driven by differing transcriptional contributions of T-ALLs and AMLs within each cluster. Further analysis of Cluster 2 revealed the surprising finding that while T-ALLs were mostly negatively enriched for lymphoid signatures, AMLs had expression patterns that resembled several lymphoidcompetent populations, including MLPs, T-oriented CD127- and B-oriented CD127+ELPs and early B-cell progenitors (Fig. 3b). Similarly, Cluster 3 AMLs showed significant enrichment for LMDP and mid-thymic signatures, while T-ALLs in the same group were more likely to resemble myeloid populations, including GMPs and MDCPs (Fig. 3c). These data suggest that interface AMLs 
a

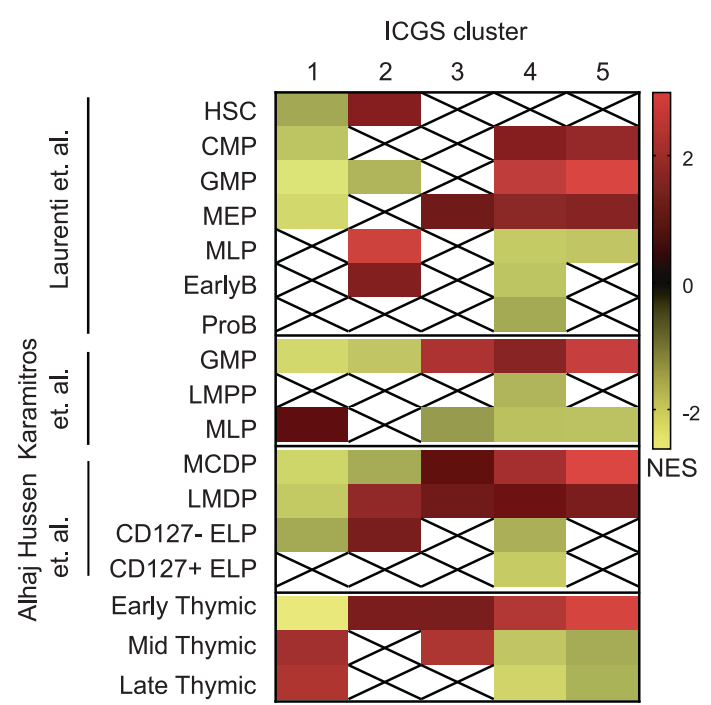

b

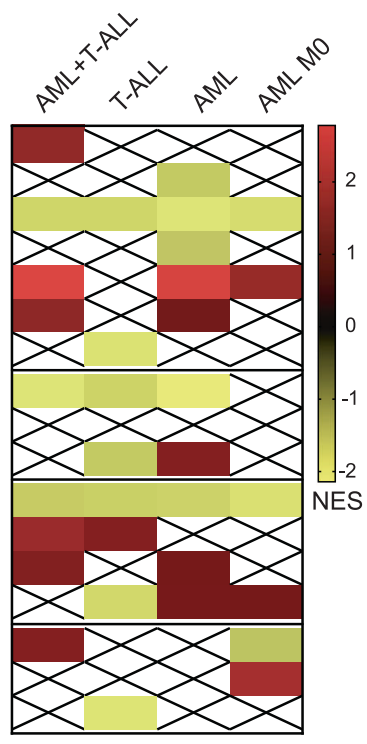

C Cluster 3

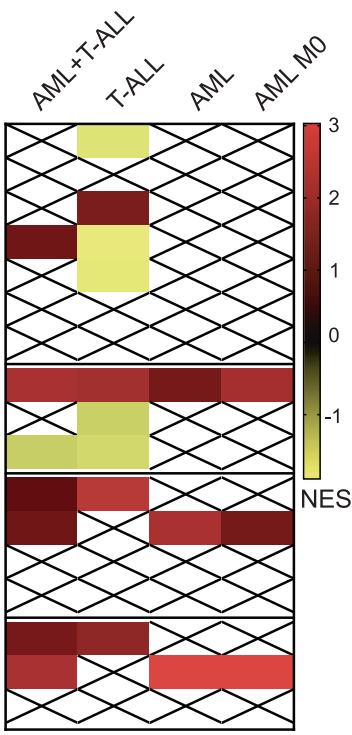

d

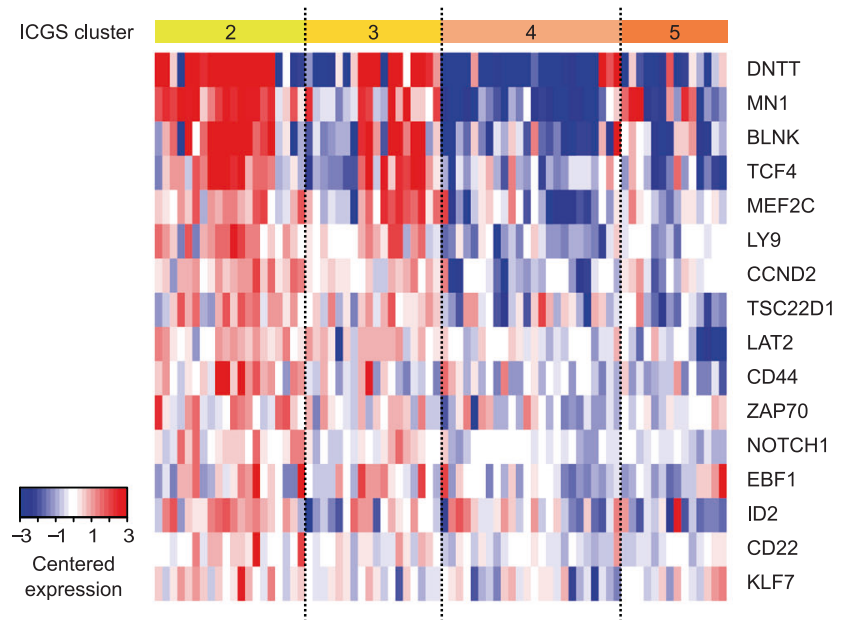

e

g

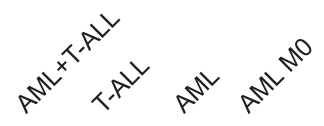

9 Cluster 3

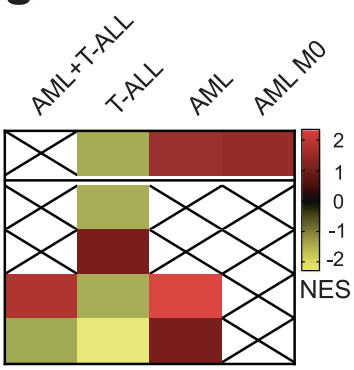

Fig. 3 Transcriptional characterization of ICGS-defined clusters. a-c: GSEAs using normal hematopoietic precursor transcriptional signatures of (a) all clusters, (b) interface Cluster 2 and (c) interface Cluster 3. Analyses restricted to either T-ALL, AML-like T-ALL, nonM0-AML and M0-AML are shown. Crossed out boxes indicate genesets that are not significantly enriched (FDR $>0.05$ ). d Comparison of expression of genes related to B-cell development in interface and noninterface AMLs. e-f Enrichment of leukemic stem cell (LSC) [34] and mixed phenotype acute leukemia (MPAL) [35, 36] transcriptional signatures by GSEA of (e) all clusters, (f) interface Cluster 2 and (g) interface Cluster 3. Analyses restricted to either T-ALL, AML-like T-ALL, non-M0-AML and M0-AML are shown. 
demonstrate significant lymphoid orientation, which can be more pronounced than the T-ALLs with which they cocluster by ICGS. Enrichment for B-lymphoid transcription was particularly evident when expression of genes related to B-cell development was compared in interface and noninterface AMLs (Fig. 3d).

\section{ICGS-defined interface AMLs transcriptionally resemble mixed phenotype leukemia}

Further GSEA revealed that interface Cluster 2 was significantly enriched for a myeloid leukemic stem cell (LSC) transcriptional signature [34], and that this enrichment was shared by both T-ALLs and AMLs in this group (Fig. 3e, f). AMLs in interface Cluster 3 (Fig. 3g), and AML-like TALLs (NES $=1.92$; FWER $=0.003$ ) were also enriched for the LSC signature, suggesting that expression of leukemia stemness genes is a common feature of IAL cases.

As interface leukemias share expression profiles with a range of progenitors of multipotent lineage capacity, we next tested whether there was any transcriptional similarity to MPALs of either T-lymphoid/myeloid (T/M MPAL) or B-lymphoid/ myeloid (B/M MPAL) phenotype in children [35] and adults [36]. We found that interface Clusters 2 and 3 were enriched for B/M MPAL and T/M MPAL signatures respectively, and that enrichment was driven by the AML cases in each group (Fig. 3f, g). Therefore, in keeping with the results observed in normal progenitor comparisons, transcriptional resemblance to the earliest stages of lymphoid orientation appears to be driven by interface AMLs rather than T-ALLs.

\section{Interface AMLs have poor outcomes}

The fact that interface AMLs exhibit markedly different transcription to other AML cases led us to speculate that these leukemias may have specific biology which in turn might affect clinical behavior. We therefore evaluated the outcome of interface AMLs in two independent studies $[44,45]$. To identify these cases, we calculated an interface AML (IAL) score based on gene expression differences between interface and non-interface AMLs in our cohort (Supplementary Methods and Supplementary Table S8). Outcome analyses revealed that AMLs with high IAL scores had significantly shorter survival in both studies (Fig. 4a, b). Within the ALFA-1701 group, these effects were mainly driven by significantly different outcomes within the ELN Intermediate Risk subgroup (Supplementary Table S9). We also found that high IAL scores predicted lack of response to gemtuzumab ozogamicin (Fig. 4c), which in keeping with our previous results [46], correlated with reduced expression of CD33 in high IAL score cases (Fig. 4d). Importantly, multivariate analysis of the ALFA-0701 cohort [45] revealed that IAL score predicted outcome independently of other prognostic variables, including cytogenetic classification, ELN subgroups and the recently-described LSC17 score [34] (Table 1). Consistent with this, our IAL signature had almost no overlap with the LSC17 signature, or the extended 48 gene signature that was reported in the same paper [34] (Supplementary Fig. S4A and B). Full comparison of clinicobiological and mutational profiles of ALFA-0701 patients with high and low IAL scores is shown in Supplementary Table S10. Finally, we evaluated whether high IAL score cases had evidence of lymphoid transcriptional activation. In keeping with our earlier results (Fig. 3), we found that high IAL score cases in both AML cohorts were significantly enriched for both MLP signatures and B-lymphoid gene expression (Supplementary Fig. S4C-G).

\section{Discussion}

In keeping with modern concepts of a hematopoietic progenitor framework that comprises a spectrum of differentiation potential, integrated transcriptional analysis of AMLs and T-ALLs revealed a continuum of leukemic developmental arrest. While AMLs and T-ALLs at either end of the spectrum were specifically enriched for the transcriptional signatures of the corresponding lineage, interface leukemias had evidence of both myeloid and lymphoid precursor gene expression, with early lymphoid signature enrichment being driven by interface AML cases. Specifically, while interface Cluster 3 AMLs had Tlymphoid transcriptional enrichment, interface Cluster 2 AMLs more closely resembled B-oriented lymphoid precursors including early B progenitors, MLPs and CD127+ ELPs [24, 38], and B/Myeloid MPAL [35, 36]. This cluster comprised a high proportion of RUNX1-mutated M0AMLs, which have previously been reported to show B-cell gene activation [47]. Overall, these results suggest that these leukemias may be more likely to arise from lymphoidoriented progenitors and/or be arrested at an early stage of lymphoid orientation (prior to CD19 expression) than is currently recognized. It should also be noted that the high proportion of IALs in this cohort reflects our deliberate selection of high proportions of immature leukemias, which exceed what is seen in routine clinical practice.

ICGS clustering presented several important differences with accepted methods of T-ALL categorization by phenotype, immunogenotype or mutational profile [9, 14, 37]. For example, the majority of immature T-ALLs defined by $T R$ rearrangement [37] $(16 / 26,61.5 \%)$ or ETP-ALL phenotype $(12 / 20,60 \%)$ [14] were in Cluster 1 , including those with JAK-STAT pathway mutations (Supplementary Table S7). In addition, IALs had low percentages of WTI 
a
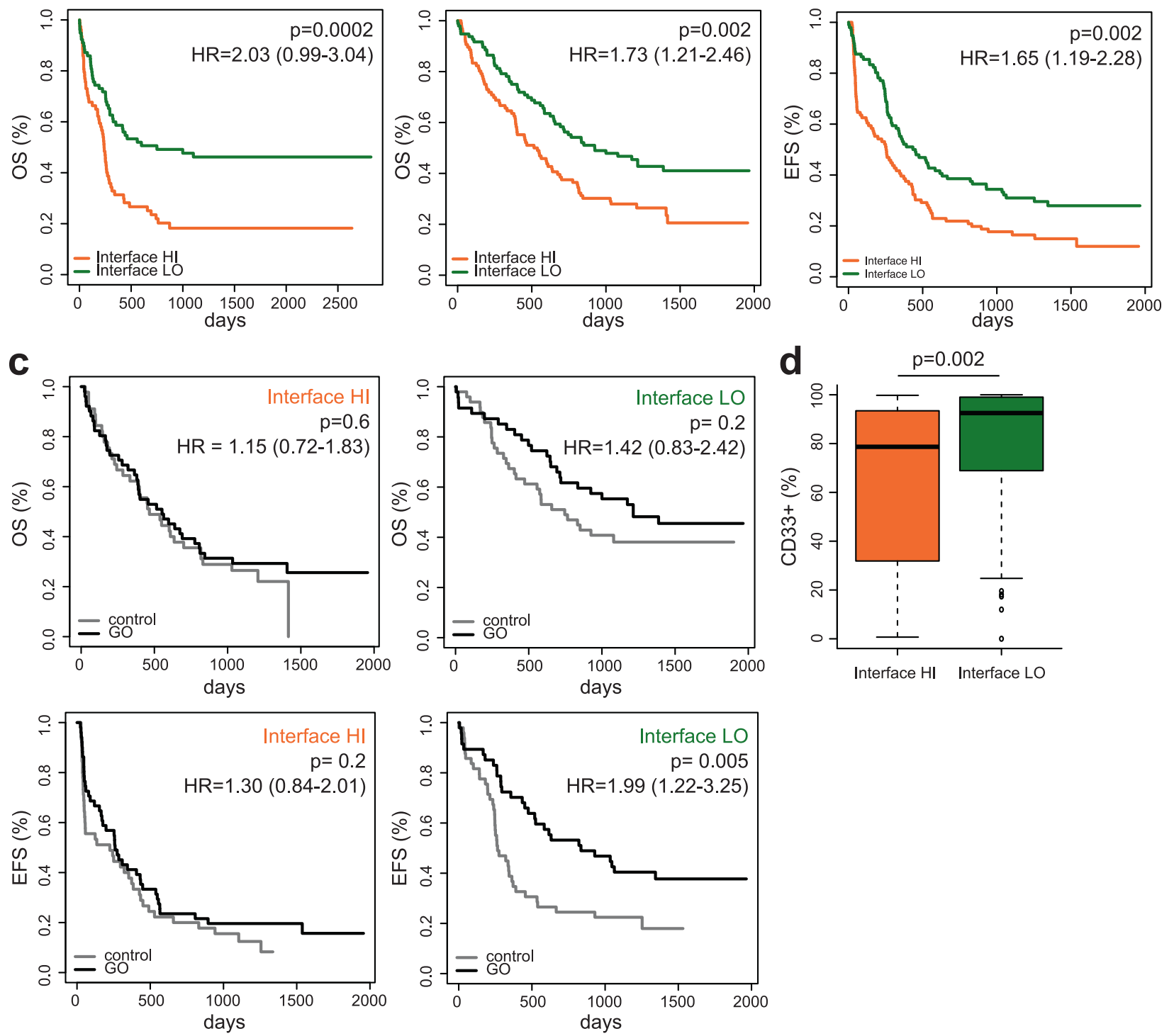

Fig. 4 Interface IALs have poor outcomes. a-c Survival comparisons of AMLs with high and low IAL scores in independent cohorts, a Metzeler et al. [44] and b ALFA-1701 [45]. OS overall survival. EFS event-free survival. Hazard ratios (HR) and 95\% Confidence Intervals for each event and $p$ values as measured by a two tailed Welch t-test

and SUZ12 mutations that are typical of ETP-ALLs [9, 10] and positive enrichment for PTEN alterations that are more frequent in mature T-ALLs [43, 48]. We also noted differences in mutational cooccurrence in these groups. While PHF6 mutations were always accompanied by NOTCHI alterations in Cluster 1, 3/5 PHF6-mutated IALs (1/3 TALL and 2/2 AML) were NOTCH1 wild-type. This pattern was also reported in MPAL [35, 49], and suggests that the leukemic phenotype of PHF6 mutation may correlate with co-expression of other oncogenes, as shown for $T L X 3$ [50]. Interestingly, PHF6 has been shown to regulate B/T lineage plasticity, at least on a $B C R-A B L$ leukemic background [51]. are indicated. c Outcome comparisons according to IAL score and treatment with gemtuzumab ozogamicin (GO) in the ALFA-1701 cohort. d Comparison of CD33 expression in IAL High and Low cases in the ALFA-1701 cohort. Boxes indicate median, interquartile range and whiskers the 95 percentile.

Interface AMLs were also not restricted to immunophenotypically immature M0 cases, since they included $29 \%$ of non-M0 AMLs.

Our description of myeloid/T-lymphoid IALs provides support for recent proposals to define acute myeloid/Tlymphoblastic leukemia (AMTL) as a distinct diagnostic entity [11], but our results also indicate that this group comprises significant molecular and lineage heterogeneity, particularly with regard to lymphoid gene expression. When applied to independent studies, our IAL score identified AMLs with similar enrichment for immature B-lymphoid signatures as interface cases in our cohort. While the IAL 
Table 1 Prognostic impact of IAL score on overall survival in the ALFA-0701 trial.

\begin{tabular}{|c|c|c|c|c|c|c|}
\hline \multirow[t]{2}{*}{ Variable } & \multicolumn{3}{|c|}{ Univariate } & \multicolumn{3}{|c|}{ Multivariate } \\
\hline & HR & $95 \%$ CI & $p$ & HR & $95 \% \mathrm{CI}$ & $p$ \\
\hline GO arm & 0.82 & $0.61-1.10$ & 0.19 & 0.85 & $0.59-1.22$ & 0.38 \\
\hline ELN risk $^{a}$ & 2.19 & $1.71-2.80$ & $<0.001$ & 1.96 & $1.35-2.75$ & $<0.001$ \\
\hline $\begin{array}{l}\text { High } \\
\text { LSC17 score }\end{array}$ & 2.45 & $1.71-3.53$ & $<0.001$ & 1.80 & $1.19-2.71$ & 0.005 \\
\hline $\begin{array}{l}\text { NPMI } \\
\text { mutation }\end{array}$ & 0.67 & $0.48-0.94$ & 0.019 & 1.47 & $0.91-2.40$ & 0.12 \\
\hline $\begin{array}{l}\text { High IAL } \\
\text { score }\end{array}$ & 1.73 & $1.21-2.46$ & 0.002 & 1.64 & $1.11-2.42$ & 0.01 \\
\hline
\end{tabular}

Covariates selected for multivariate analyses were selected based on the results of univariate analyses (full results in Supplementary Table S11), with additional retention of GO (gemtuzumab ozogamicin) treatment arm.

$H R$ hazard ratio.

${ }^{a}$ According to the ELN-2010 classification [56] that was in use during the ALFA-0701 trial, both Intermediate-1 and Intermediate-2 subgroups were considered in a single intermediate subgroup. 16 nonclassifiable patients were excluded from the analysis.

${ }^{\mathrm{b}}$ The LSC17 score was described in $\mathrm{Ng}$ et al. [34]. Statistically significant differences are shown in bold.

score was not designed to predict prognosis, it is striking that these transcriptional patterns correlated with poor response to AML treatment regimens. RUNX1-mutated AML-M0 cases in our cohort showed B-lymphoid identity, which is consistent with previous reports [47]. Intriguingly, RUNX1-mutated AMLs have recently been shown to be sensitive to glucocorticoids [52], which form the backbone of ALL induction treatment. Our findings therefore suggest that the poor response of these cases to AML therapy in both adults [53] and children [54] might be improved by better treatment allocation, and would plead against the recent provisional classification of RUNXI-mutated AMLM0 with AML [2]. Finally, we hope that these data will provide further impetus to include these and other IALs in shared myeloid/lymphoid protocols that might provide better treatment options for patients with these poor-risk leukemias [55].

\section{Data availability}

Gene expression data reported here is available at GEO as superseries GSE131207 (normal thymic populations dataset: GSE131180; AMLs/T-ALLs dataset: GSE131184). Other experimental methods are described in the Supplemental Data.

Acknowledgements The Necker laboratory was supported by the Association Laurette Fugain, La ligue contre le Cancer and the INCa 2007 (DM/FC/sl/RT07) and CARAMELE Translational Research and
$\mathrm{PhD}$ programs and INCa/AP-HP genetic Plateforme de Ressources Biologiques (PRB). Certain AL samples were collected within the MILE program. JB was supported by a Kay Kendall Leukaemia Fund Intermediate Research Fellowship and by the National Children's Research Centre, Children's Health Ireland at Crumlin, Dublin. Work in the Laurenti laboratory in Cambridge was supported by a Wellcome/Royal Society Sir Henry Dale Fellowship to EL (Grant number 107630/Z/15/Z), the European Hematology Association, BBSRC and by core funding from Wellcome (Grant number 203151/Z/16/Z) and MRC to the Wellcome-MRC Cambridge Stem Cell Institute. AK was funded by a National Science Centre, Poland research grant (2017/01/ X/ST6/01329). We thank Jinyan Huang at the Shanghai Institute of Hematology $(\mathrm{SIH})$ for help in retrieving datasets from the Chinese Leukemia Genotype-Phenotype Archive, as reported by Chen et al. [33]. We confirm that those who carried out the original analysis and collection of these data at SIH bear no responsibility for our further analysis and interpretation of such data. We would also like to thank Koichi Takahashi for annotation of samples from GSE11360, and the Plateforme Biopuces et Séquençage of the IGBMC (Strasbourg) for performance of expression microarray experiments.

\section{Compliance with ethical standards}

Conflict of interest The authors declare no conflict of interest.

Publisher's note Springer Nature remains neutral with regard to jurisdictional claims in published maps and institutional affiliations.

Open Access This article is licensed under a Creative Commons Attribution 4.0 International License, which permits use, sharing, adaptation, distribution and reproduction in any medium or format, as long as you give appropriate credit to the original author(s) and the source, provide a link to the Creative Commons license, and indicate if changes were made. The images or other third party material in this article are included in the article's Creative Commons license, unless indicated otherwise in a credit line to the material. If material is not included in the article's Creative Commons license and your intended use is not permitted by statutory regulation or exceeds the permitted use, you will need to obtain permission directly from the copyright holder. To view a copy of this license, visit http://creativecommons. org/licenses/by/4.0/.

\section{References}

1. Chiaretti S, Gianfelici V, O'Brien SM, Mullighan CG. Advances in the genetics and therapy of acute lymphoblastic leukemia. Am Soc Clin Oncol Educ Book. 2016;35:e314-22.

2. Arber DA, Orazi A, Hasserjian R, Thiele J, Borowitz MJ, Le Beau MM, et al. The 2016 revision to the World Health Organization classification of myeloid neoplasms and acute leukemia. Blood. 2016;127:2391-405.

3. Rubnitz JE, Onciu M, Pounds S, Shurtleff S, Cao X, Raimondi $\mathrm{SC}$, et al. Acute mixed lineage leukemia in children: the experience of St Jude Children's Research Hospital. Blood. 2009;113:5083-9.

4. Kurosawa S, Toya T, Kishida Y, Nagata A, Yamada Y, Konishi $\mathrm{T}$, et al. Outcome of patients with acute undifferentiated leukemia after allogeneic hematopoietic stem cell transplantation. Leuk Lymphoma. 2018;59:3006-9.

5. Wolach O, Stone RM. How I treat mixed-phenotype acute leukemia. Blood. 2015;125:2477-85.

6. Ferrando AA, Neuberg DS, Staunton J, Loh ML, Huard C, Raimondi SC, et al. Gene expression signatures define novel 
oncogenic pathways in $\mathrm{T}$ cell acute lymphoblastic leukemia. Cancer Cell. 2002;1:75-87.

7. Belver L, Ferrando A. The genetics and mechanisms of $\mathrm{T}$ cell acute lymphoblastic leukaemia. Nat Rev Cancer. 2016;16:494-507.

8. Bond J, Marchand T, Touzart A, Cieslak A, Trinquand A, Sutton L, et al. An early thymic precursor phenotype predicts outcome exclusively in HOXA-overexpressing adult T-cell acute lymphoblastic leukemia: a Group for Research in Adult Acute Lymphoblastic Leukemia study. Haematologica. 2016;101:732-40.

9. Zhang J, Ding L, Holmfeldt L, Wu G, Heatley SL, Payne-Turner $\mathrm{D}$, et al. The genetic basis of early T-cell precursor acute lymphoblastic leukaemia. Nature. 2012;481:157-63.

10. Bond J, Graux C, Lhermitte L, Lara D, Cluzeau T, Leguay T, et al. Early response-based therapy stratification improves survival in adult early thymic precursor acute lymphoblastic leukemia: a group for research on adult acute lymphoblastic leukemia study. J Clin Oncol. 2017;35:2683-91.

11. Gutierrez A, Kentsis A. Acute myeloid/T-lymphoblastic leukaemia (AMTL): a distinct category of acute leukaemias with common pathogenesis in need of improved therapy. Br J Haematol. 2018;180:919-24.

12. Neumann M, Greif PA, Baldus CD. Mutational landscape of adult ETP-ALL. Oncotarget. 2013;4:954-5.

13. Grossmann V, Haferlach C, Weissmann S, Roller A, Schindela S, Poetzinger F, et al. The molecular profile of adult T-cell acute lymphoblastic leukemia: mutations in RUNX1 and DNMT3A are associated with poor prognosis in T-ALL. Genes Chromosomes Cancer. 2013;52:410-22.

14. Coustan-Smith E, Mullighan CG, Onciu M, Behm FG, Raimondi SC, Pei D, et al. Early T-cell precursor leukaemia: a subtype of very high-risk acute lymphoblastic leukaemia. Lancet Oncol. 2009;10:147-56.

15. Gougounon A, Abahssain H, Rigollet L, Elhamri M, Tigaud I, Chelghoum Y, et al. Minimally differentiated acute myeloid leukemia (FAB AML-M0): prognostic factors and treatment effects on survival-a retrospective study of 42 adult cases. Leuk Res. 2011;35:1027-31.

16. Wood BL, Winter SS, Dunsmore KP, Devidas M, Chen S, Asselin $\mathrm{B}$, et al. T-lymphoblastic leukemia (T-ALL) shows excellent outcome, lack of significance of the early thymic precursor (ETP) immunophenotype, and validation of the prognostic value of endinduction minimal residual disease (MRD) in children's oncology group (COG) study AALL0434. Blood. 2014;124:1.

17. Walter RB, Othus M, Burnett AK, Lowenberg B, Kantarjian HM, Ossenkoppele GJ, et al. Significance of FAB subclassification of "acute myeloid leukemia, NOS" in the 2008 WHO classification: analysis of 5848 newly diagnosed patients. Blood. 2013; 121:2424-31.

18. Barbaric D, Alonzo TA, Gerbing RB, Meshinchi S, Heerema NA, Barnard DR, et al. Minimally differentiated acute myeloid leukemia (FAB AML-M0) is associated with an adverse outcome in children: a report from the Children's Oncology Group, studies CCG-2891 and CCG-2961. Blood. 2007;109:2314-21.

19. Laurenti E, Gottgens B. From haematopoietic stem cells to complex differentiation landscapes. Nature. 2018;553:418-26.

20. Belluschi S, Calderbank EF, Ciaurro V, Pijuan-Sala B, Santoro A, Mende N, et al. Myelo-lymphoid lineage restriction occurs in the human haematopoietic stem cell compartment before lymphoidprimed multipotent progenitors. Nat Commun. 2018;9:4100.

21. Doulatov S, Notta F, Laurenti E, Dick JE. Hematopoiesis: a human perspective. Cell Stem Cell. 2012;10:120-36.

22. Goardon N, Marchi E, Atzberger A, Quek L, Schuh A, Soneji S, et al. Coexistence of LMPP-like and GMP-like leukemia stem cells in acute myeloid leukemia. Cancer Cell. 2011;19:138-52.
23. Karamitros D, Stoilova B, Aboukhalil Z, Hamey F, Reinisch A, Samitsch M, et al. Single-cell analysis reveals the continuum of human lympho-myeloid progenitor cells. Nat Immunol. 2018;19:85-97.

24. Alhaj Hussen K, Vu Manh TP, Guimiot F, Nelson E, Chabaane E, Delord $\mathrm{M}$, et al. Molecular and functional characterization of lymphoid progenitor subsets reveals a bipartite architecture of human lymphopoiesis. Immunity. 2017;47:680-96 e8.

25. Kohn LA, Hao QL, Sasidharan R, Parekh C, Ge S, Zhu Y, et al. Lymphoid priming in human bone marrow begins before expression of CD10 with upregulation of L-selectin. Nat Immunol. 2012;13:963-71.

26. Hao QL, George AA, Zhu J, Barsky L, Zielinska E, Wang X, et al. Human intrathymic lineage commitment is marked by differential CD7 expression: identification of CD7- lympho-myeloid thymic progenitors. Blood. 2008;111:1318-26.

27. Luc S, Luis TC, Boukarabila H, Macaulay IC, Buza-Vidas N, Bouriez-Jones $\mathrm{T}$, et al. The earliest thymic $\mathrm{T}$ cell progenitors sustain B cell and myeloid lineage potential. Nat Immunol. 2012;13:412-9.

28. Booth CAG, Barkas N, Neo WH, Boukarabila H, Soilleux EJ, Giotopoulos G, et al. Ezh2 and Runx1 mutations collaborate to initiate lympho-myeloid leukemia in early thymic progenitors. Cancer Cell. 2018;33:274-91 e8.

29. Soulier J, Clappier E, Cayuela JM, Regnault A, Garcia-Peydro M, Dombret H, et al. HOXA genes are included in genetic and biologic networks defining human acute T-cell leukemia (T-ALL). Blood. 2005; 106:274-86.

30. Homminga I, Pieters R, Langerak AW, de Rooi JJ, Stubbs A, Verstegen $\mathrm{M}$, et al. Integrated transcript and genome analyses reveal NKX2-1 and MEF2C as potential oncogenes in T cell acute lymphoblastic leukemia. Cancer Cell. 2011;19:484-97.

31. Olsson A, Venkatasubramanian M, Chaudhri VK, Aronow BJ, Salomonis N, Singh H, et al. Single-cell analysis of mixed-lineage states leading to a binary cell fate choice. Nature. 2016;537:698-702.

32. Liu Y, Easton J, Shao Y, Maciaszek J, Wang Z, Wilkinson MR, et al. The genomic landscape of pediatric and young adult Tlineage acute lymphoblastic leukemia. Nat Genet. 2017;49:1211-8.

33. Chen B, Jiang L, Zhong ML, Li JF, Li BS, Peng LJ, et al. Identification of fusion genes and characterization of transcriptome features in T-cell acute lymphoblastic leukemia. Proc Natl Acad Sci USA. 2018;115:373-8.

34. Ng SW, Mitchell A, Kennedy JA, Chen WC, McLeod J, Ibrahimova N, et al. A 17-gene stemness score for rapid determination of risk in acute leukaemia. Nature. 2016;540:433-7.

35. Alexander TB, Gu Z, Iacobucci I, Dickerson K, Choi JK, Xu B, et al. The genetic basis and cell of origin of mixed phenotype acute leukaemia. Nature. 2018;562:373-9.

36. Takahashi K, Wang F, Morita K, Yan Y, Hu P, Zhao P, et al. Integrative genomic analysis of adult mixed phenotype acute leukemia delineates lineage associated molecular subtypes. Nat Commun. 2018;9:2670.

37. Asnafi V, Beldjord K, Boulanger E, Comba B, Le Tutour P, Estienne MH, et al. Analysis of TCR, pT alpha, and RAG-1 in Tacute lymphoblastic leukemias improves understanding of early human T-lymphoid lineage commitment. Blood. 2003; 101:2693-703.

38. Laurenti E, Doulatov S, Zandi S, Plumb I, Chen J, April C, et al. The transcriptional architecture of early human hematopoiesis identifies multilevel control of lymphoid commitment. Nat Immunol. 2013;14:756-63.

39. Cante-Barrett K, Mendes RD, Li Y, Vroegindeweij E, PikeOverzet K, Wabeke T, et al. Loss of CD44(dim) expression from 
early progenitor cells marks T-cell lineage commitment in the human thymus. Front Immunol. 2017;8:32.

40. Kim R, Boissel N, Touzart A, Leguay T, Thonier F, Thomas X, et al. Adult T-cell acute lymphoblastic leukemias with IL7R pathway mutations are slow-responders who do not benefit from allogeneic stem-cell transplantation. Leukemia. 2020;34:1730-40.

41. Bond J, Touzart A, Lepretre S, Graux C, Bargetzi M, Lhermitte L, et al. DNMT3A mutation is associated with increased age and adverse outcome in adult T-acute lymphoblastic leukemia. Haematologica. 2019;104:1617-25.

42. Neumann M, Heesch S, Schlee C, Schwartz S, Gokbuget N, Hoelzer D, et al. Whole-exome sequencing in adult ETP-ALL reveals a high rate of DNMT3A mutations. Blood 2013;121:4749-52.

43. Trinquand A, Tanguy-Schmidt A, Ben Abdelali R, Lambert J, Beldjord K, Lengline E, et al. Toward a NOTCH1/FBXW7/RAS/ PTEN-based oncogenetic risk classification of adult T-cell acute lymphoblastic leukemia: a Group for Research in Adult Acute Lymphoblastic Leukemia study. J Clin Oncol. 2013;31:4333-42.

44. Metzeler KH, Hummel M, Bloomfield CD, Spiekermann K, Braess J, Sauerland MC, et al. An 86-probe-set gene-expression signature predicts survival in cytogenetically normal acute myeloid leukemia. Blood. 2008;112:4193-201.

45. Castaigne S, Pautas C, Terre C, Raffoux E, Bordessoule D, Bastie $\mathrm{JN}$, et al. Effect of gemtuzumab ozogamicin on survival of adult patients with de-novo acute myeloid leukaemia (ALFA0701): a randomised, open-label, phase 3 study. Lancet. 2012;379:1508-16

46. Olombel G, Guerin E, Guy J, Perrot JY, Dumezy F, de Labarthe A, et al. The level of blast CD33 expression positively impacts the effect of gemtuzumab ozogamicin in patients with acute myeloid leukemia. Blood. 2016;127:2157-60.

47. Silva FP, Swagemakers SM, Erpelinck-Verschueren C, Wouters BJ, Delwel R, Vrieling H, et al. Gene expression profiling of minimally differentiated acute myeloid leukemia: M0 is a distinct entity subdivided by RUNX1 mutation status. Blood. 2009;114:3001-7.
48. Tesio M, Trinquand A, Ballerini P, Hypolite G, Lhermitte L, Petit A, et al. Age-related clinical and biological features of PTEN abnormalities in T-cell acute lymphoblastic leukaemia. Leukemia. 2017;31:2594-600.

49. Mi X, Griffin G, Lee W, Patel S, Ohgami R, Ok CY, et al. Genomic and clinical characterization of $\mathrm{B} / \mathrm{T}$ mixed phenotype acute leukemia reveals recurrent features and T-ALL like mutations. Am J Hematol. 2018;93:1358-67.

50. McRae HM, Garnham AL, Hu Y, Witkowski MT, Corbett MA, Dixon MP, et al. PHF6 regulates hematopoietic stem and progenitor cells and its loss synergizes with expression of TLX3 to cause leukemia. Blood. 2019;133:1729-41.

51. Soto-Feliciano YM, Bartlebaugh JME, Liu Y, Sanchez-Rivera FJ, Bhutkar A, Weintraub AS, et al. PHF6 regulates phenotypic plasticity through chromatin organization within lineage-specific genes. Genes Dev. 2017;31:973-89.

52. Simon L, Lavallee VP, Bordeleau ME, Krosl J, Baccelli I, Boucher $\mathrm{G}$, et al. Chemogenomic Landscape of RUNX1-mutated AML Reveals Importance of RUNX1 Allele Dosage in Genetics and Glucocorticoid Sensitivity. Clin Cancer Res. 2017;23:6969-81.

53. Gaidzik VI, Teleanu V, Papaemmanuil E, Weber D, Paschka P, Hahn J, et al. RUNX1 mutations in acute myeloid leukemia are associated with distinct clinico-pathologic and genetic features. Leukemia. 2016;30:2160-8.

54. Duployez N, Preudhomme C, Cheok M. A 17-gene-expression profile to improve prognosis prediction in childhood acute myeloid leukemia. Oncotarget. 2018;9:33869-70.

55. Kohlmann A, Kipps TJ, Rassenti LZ, Downing JR, Shurtleff SA, Mills KI, et al. An international standardization programme towards the application of gene expression profiling in routine leukaemia diagnostics: the Microarray Innovations in LEukemia study prephase. Br J Haematol. 2008;142:802-7.

56. Dohner H, Estey EH, Amadori S, Appelbaum FR, Buchner T, Burnett AK, et al. Diagnosis and management of acute myeloid leukemia in adults: recommendations from an international expert panel, on behalf of the European LeukemiaNet. Blood. 2010;115:453-74. 